\title{
Protective effects of exogenous gangliosides on ROS-induced changes in human spermatozoa
}

\author{
Mirjana Gavella and Vaskresenija Lipovac
}

This article summarizes the available evidence on the efficacy of gangliosides to reduce the degree of reactive oxygen species (ROS)-mediated damage. The antioxidative efficacy of exogenous gangliosides in protecting different cells encouraged us to examine their ability to protect human spermatozoa. Gangliosides are sialic acid-containing glycosphingolipids with strong amphiphilic character due to the bulky headgroup made of several sugar rings with sialic acid residues and the double-tailed hydrophobic lipid moiety. The amphiphilicity of gangliosides allows them to exist as micelles in aqueous media when they are present at a concentration above their critical micellar concentration. The protective effect of ganglioside micelles on spermatozoa is believed to stem from their ability to scavenge free radicals and prevent their damaging effects. In our study, we particularly focused our attention on the protective effect of ganglioside micelles on DNA in human spermatozoa exposed to cryopreservation. The results indicate that ganglioside micelles can modulate the hydrophobic properties of the sperm membrane to increase tolerance to DNA fragmentation, thus protecting the DNA from cryopreservation-induced damage. Further actions of ganglioside micelles, which were documented by biochemical and biophysical studies, included (i) the modulation of superoxide anion generation by increasing the diffusion barrier for membrane events responsible for signal translocation to the interior of the cell; (ii) the inhibition of iron-catalysed hydroxyl radical formation due to the iron chelation potential of gangliosides; and (iii) inhibition of hydrogen peroxide diffusion across the sperm membrane.

Asian Journal of Andrology (2013) 15, 375-381; doi:10.1038/aja.2012.144; published online 18 March 2013

Keywords: exogenous gangliosides; oxidative stress; protection; spermatozoa

\section{INTRODUCTION}

To understand the causes of reduced fertilising potential, the mechanisms responsible for impaired sperm function need to be identified. An increasing body of evidence suggests that oxidative stress is a dominant feature in the aetiology of male infertility. Sperm damage is a consequence of a variety of processes that occur during the life span of human spermatozoa and induce the generation of excessive oxygenderived reactants, such as superoxide anion $\left(\mathrm{O}_{2}{ }^{-}\right)$, hydrogen peroxide $\left(\mathrm{H}_{2} \mathrm{O}_{2}\right)$ and a highly toxic hydroxyl radical $(\mathrm{OH} \cdot) .{ }^{1-4}$

Spermatozoa are particularly susceptible to damage by processes involving free radicals for several reasons. The sperm membrane is rich in polyunsaturated fatty acids, which provide a sufficient fluidity of the sperm membrane to allow it to participate in the membrane fusion events that characterize fertilisation. ${ }^{5,6}$ Polyunsaturated fatty acids are particularly prone to oxidative attack, as the presence of a double bond weakens $\mathrm{C}-\mathrm{H}$ bonds on adjacent carbon atoms and facilitates hydrogen abstraction, which initiates a lipid peroxidation cascade. $^{6-8}$ The limited enzymatic antioxidative defence system in spermatozoa also increases their susceptibility to oxidative stress.

There are two main mechanisms by which reactive oxygen species (ROS) cause infertility. First, ROS damage the sperm membrane, which in turn, reduces sperm motility and their ability to fuse with the oocyte. Second, ROS directly damage sperm DNA, compromising the paternal genomic contribution to the embryo. ${ }^{4,9}$ Recently, studies have focused on assessing the effectiveness of in vivo and in vitro supplementation with different antioxidants in an attempt to protect spermatozoa exposed to oxidative stress. ${ }^{10-14}$ A beneficial effect of the in vitro supplementation of different natural or chemically synthesized antioxidant compounds on human spermatozoa has been observed; however, their effect has also been shown to decrease at inadequate concentrations and, even worse, to behave as pro-oxidants. ${ }^{11,12,15,16}$

Herein, we discuss the protective role of exogenous gangliosides against ROS-induced changes based on our studies of human spermatozoa, leukocytes and cell-free systems. Of particular interest are differences between the effects of various types of gangliosides on the lipid peroxidation level, superoxide anion production ${ }^{17-20}$ and $\mathrm{H}_{2} \mathrm{O}_{2}$ and cryopreservation-induced DNA damage of human spermatozoa. $^{21,22}$

\section{GANGLIOSIDES}

Gangliosides, which are sialic acid-containing glycosphingolipids, are expressed in the outer leaflet of the plasma membrane of all mammalian cell membranes, including spermatozoa, ${ }^{23,24}$ and are particularly abundant in neuronal cell membranes. ${ }^{25}$ A large number of gangliosides have been isolated from the brains of various organisms, but only four structures, GM1, GD1a, GD1b and GT1b, constitute more than $90 \%$ of gangliosides in the brains of various mammalian species. ${ }^{26}$ Depending on the number of sialic acid residues on the inner 
galactose, gangliosides belong to either $b$-ganglio-series (GD1b and GT1b) or a-ganglio-series (GM1 and GD1a). ${ }^{27}$ Studies of their molecular structures have shown that the number of sugar units and the position and linkage type of sialic acids distinguish each ganglioside ${ }^{28}$ and affect their physicochemical properties. ${ }^{29}$ Thus, the disialoganglioside GD1b has two sialic acids linked to the inner galactose, whereas GD1a has one sialic acid on the inner galactose and another on the terminal galactose. Figure 1 shows the structure of the monoand trisialogangliosides GM1 and GT1b. Both gangliosides have the same hydrophobic ceramide and differently structured hydrophilic parts made of several sugar rings, some of which are sialic acid residues. GM1 has one sialic acid residue on the inner galactose, while GT1b has three sialic acid residues, two linked to the inner galactose and one on the terminal galactose. ${ }^{27}$

Gangliosides are involved in the regulation of a wide range of cellular functions, serving as antigens, mediators of cell adhesion and modulators of signal transduction. ${ }^{30}$ Gangliosides have been shown to control cellular growth and differentiation and to play important roles in the immune defence system. Gangliosides are not distributed uniformly in the plasma membrane, and they are identified as constituents of the raft microdomain, where they play important roles in the early events of signal transduction by arranging the environment of ligand-receptor interactions. ${ }^{25,31,32}$

In addition to these naturally occurring, endogenous manifestations, exogenously added gangliosides have been shown to affect cell properties and biological events, including reducing the ROS associated with oxidative stress. Due to the importance of gangliosides in the development and function of the nervous system, the majority of studies have focused on the protective effects of gangliosides against neuronal injuries.

Evidence that gangliosides might have an antioxidative capability in cultures of other cell types has accumulated over the past decades based on the observed protection against reactive oxygen formation in isolated heart, ${ }^{33}$ epithelial lens and retinal lens cells ${ }^{34}$ and hepatocytes. ${ }^{35}$ Their mode of action varies in different cell types, including altering the activity of several enzymes and specific proteins, ${ }^{36-40}$ membrane stabilisation and fluidity, ${ }^{34,35}$ scavenging hydroxyl radicals. ${ }^{41}$

Even if there were a consensus on the protective effects of gangliosides against cellular damage, the mechanism(s) underlying these effects and the models in which gangliosides are protective would still require precise evaluation. If oxidative stress and apoptosis are chemically induced in cultures of hepatocytes and rat liver epithelial cells,

Ganglioside GM1
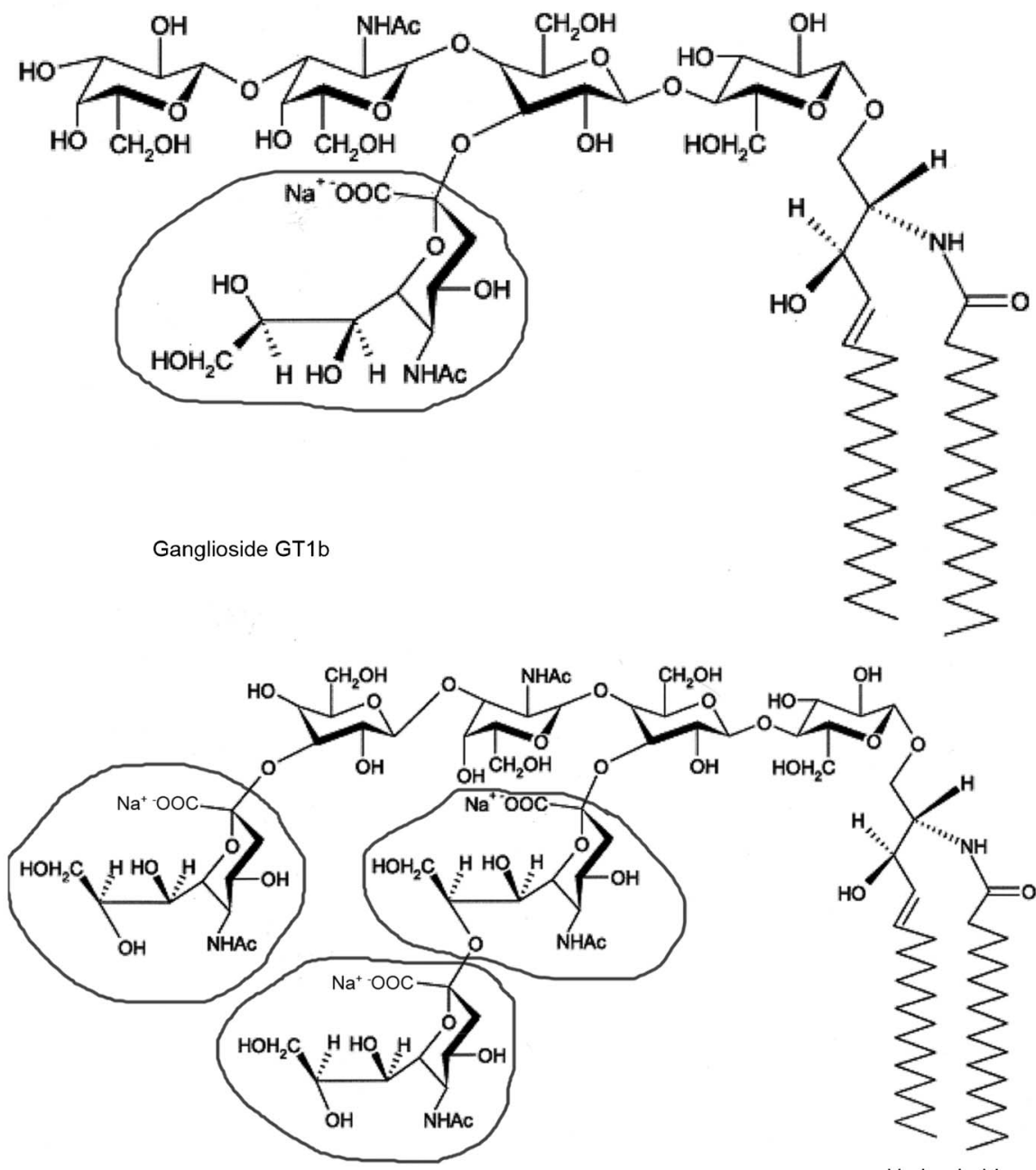

Hydrophilic oligosaccharide part

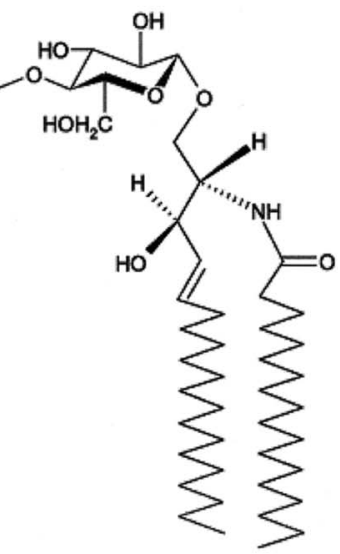

Hydrophobic ceramide part

Figure 1 Structure of the monoganglioside GM1 and the trisialoganglioside GT1b. The chemical structural formula in the circle represents the sialic acid 
exposure to the monosialoganglioside GM1 inhibits ROS and lipid peroxidation (LPO) production in the cells. ${ }^{35}$ In vivo, GT1b prevents chemically induced DNA damage in mice. ${ }^{41}$ There is evidence that the antioxidative properties of GM1 are dependent on its effect on iron ion exchange ${ }^{35,42}$ because iron ions are involved in ROS and LPO product formation in many cell types.

\section{GANGLIOSIDES AND SPERMATOZOA}

In earlier investigations conducted to identify the types of gangliosides in the sperm membrane, endogenous mono-, di- and trisialogangliosides (GM3, GM1, GD1a, GD1b and GT1b) have been observed in animal $^{23}$ and human spermatozoa ${ }^{24}$ at low concentrations of approximately $1 \mu \mathrm{g}-5 \mu \mathrm{g}$ per 100 million spermatozoa, ${ }^{43}$ although their exact function has not been completely elucidated.

Some progress was achieved over the past decade. The endogenous ganglioside GM1 has been found in the plasma membrane overlying the acrosome of the sperm head in several mammalian species ${ }^{44}$ or over the whole sperm surface in human spermatozoa. ${ }^{45} \mathrm{GM} 1$ is known to influence several signalling pathways in somatic cells and its importance in sperm functional maturation, such as capacitation, has been recently established. It has been localized in lipid raft microdomains, which serve as platforms for the assembly of key recognition molecules on the sperm surface during capacitation. ${ }^{44-47}$ In animal sperm, increasing evidence suggests that capacitation is regulated by the redistribution of GM1 on the plasma membrane. ${ }^{48}$ For example, when murine sperm migrate to the uterine cavity, GM1 shifts from the postacrosomal region and disappears from the sperm head in the oviduct, at which time, spermatozoa can undergo the acrosome reaction. ${ }^{49}$

While the role of endogenous gangliosides in sperm signalling systems has been described in the literature, no information on the protective role of exogenously supplied gangliosides against ROS-induced changes in human spermatozoa was available. Our biochemical and biophysical studies have provided evidence of the ability of gangliosides to protect human sperm against LPO, superoxide and hydroxyl formation and ROS-induced DNA damage. ${ }^{17-22}$

The protective effect of gangliosides on the lipid peroxidation level As a model for studying the ability of different gangliosides to counteract lipid peroxidation in human spermatozoa, we used ferrous/ascorbate-induced LPO. In this system, LPO is not initiated through hydroxyl radical generation, but rather through the ferrous ion-catalysed breakdown of pre-existing lipid hydroperoxide in the sperm plasma membrane and the subsequent propagation of the lipid peroxidation chain reaction via alkoxyl radical generation. ${ }^{50-53}$ According to our experiments, the monosialoganglioside GM1 and the disialoganglioside GDla have no effect on LPO propagation. However, the generation of malondialdehyde as a breakdown product of lipid peroxide decompensation was significantly decreased $(P<0.01)$ in the presence of the disialoganglioside GD1b and the trisialoganglioside GT1b. ${ }^{17}$ This could be explained by the specific position of sialic acid residues in the gangliosides of the $b$-ganglioseries (two sialic acids linked to the inner galactose) ) $^{27-29}$ and their ability to chelate ferrous ions and inhibit the propagation of the lipid peroxidative chain reaction and malondialdehyde production.

Determining the malondialdehyde level only provides an indirect measure of the lipid peroxidation process, without subcellular resolution of membrane changes. ${ }^{54} \mathrm{~A}$ few reports have been published on the application of physical methods for investigating membrane fluidity in human spermatozoa ${ }^{55-57}$ and on the ability of some agents to prevent changes in membrane fluidity induced by free radical attack. ${ }^{58,59}$
Several agents are known to act as stabilizers or protectors of the cell membrane due to their inhibition of lipid peroxidation. ${ }^{60,61}$

To investigate the effect of the ganglioside GT1b on the changes in sperm membrane fluidity induced by external oxidative stimuli, we used electron paramagnetic resonance (EPR) spectroscopy. The spectral analysis obtained by spin label 5-doxylstearic acid nitroxide, for which the paramagnetic centre resides are near the lipid/water interface in the sperm membrane, ${ }^{56}$ indicated that the oxidation-induced changes were significantly suppressed $(P<0.001)$ in the presence of the trisialoganglioside GT1b. ${ }^{18,19}$ Furthermore, when using electron spin resonance spectroscopy with the DMPO spin trap, a difference was detected in the effect of gangliosides on changes in the molecular ordering of the sperm membrane upon iron-induced oxidation. ${ }^{19}$ The presence of free iron in human seminal plasma is sufficient to promote a lipid peroxidation cascade in spermatozoa. ${ }^{62}$ Iron is believed to be one of the main mediators of the Fenton reaction and therefore to contribute to the formation of hydroxyl radicals. ${ }^{4}$ Our EPR study of ganglioside antioxidant properties in a Fenton-type reaction demonstrated the ability of ganglioside micelles to bind oppositely charged ferrous ions, thus reducing their concentrations and consequently inhibiting hydroxyl radical formation (Figure 2). ${ }^{20}$ Both biophysical and biochemical analyses have therefore clearly shown the ability of gangliosides to decrease the level of iron-induced lipid peroxidation in human spermatozoa.

\section{The protective effect of gangliosides against superoxide anion $\left(\mathrm{O}_{2} \cdot{ }^{-}\right)$production}

There are few reports concerning the beneficial effect of exogenously applied gangliosides on ROS generation. Gangliosides have been found to directly scavenge superoxide anions in the rat heart and to modulate signal transduction in rat brain synaptosomes. ${ }^{33,37,63}$ Our study on human spermatozoa revealed that the exogenously added diand trisialogangliosides GD1b and GT1b could reduce the level of superoxide anions generated by phorbol,12-myristate acetate (PMA)-stimulated human spermatozoa. ${ }^{17}$ Our recent research on ROS production in PMA-activated human leukocytes modulated by gangliosides demonstrated that exogenous GM1 and GT1b micelles could decrease the extracellular production of superoxide radicals in a

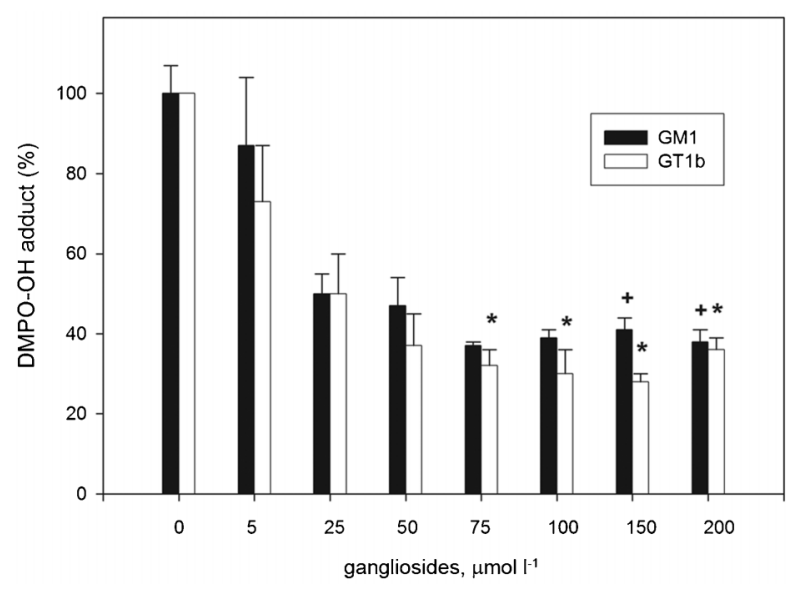

Figure 2 The EPR study of DMPO-OH spin adduct formation in a Fenton-type reaction in the presence and absence of different concentrations of GM1 ( $\boldsymbol{\square}$ ) and GT1b ( $\square)$. The data are normalized with respect to the EPR intensity of control samples without gangliosides. Significant differences are ${ }^{+} P<0.05$ for $\mathrm{GM} 1$ and $* P<0.05$ for GT1b compared to control values. EPR, electron paramagnetic resonance. 
concentration- and structure-dependent manner. The observed effect can be attributed to the ability of ganglioside micelles attached to the cell surface to provide a diffusion barrier and consequently delay membrane events responsible for superoxide anion production (Figure 3). ${ }^{64}$ The presence of leukocytes on the sperm surface and the similarity of their cellular mechanisms for superoxide generation to those of activated spermatozoa could explain the decrease in superoxide anion generation in spermatozoa. ${ }^{65,66}$ It is believed that leukocytes and spermatozoa share a similar mechanism for ROS generation: an NADPH oxidase located in or near the cell membrane. ${ }^{67}$ Although controversy exists regarding the form of this enzyme, ${ }^{68}$ exogenous gangliosides undeniably decrease the extracellular production of superoxide radicals.

\section{The protective effect of gangliosides on DNA damage}

Many studies have clearly shown that excessive ROS generation affects the DNA integrity of spermatozoa. In vitro experiments in which spermatozoa were exposed to initiators of oxidative stress such as $\mathrm{H}_{2} \mathrm{O}_{2}$ revealed a significant increase in DNA damage in the form of either single- and double-strand breaks or DNA modification products. $^{69-71}$ Our investigations have provided evidence showing that DNA fragmentation in human spermatozoa after in vitro exposure to $\mathrm{H}_{2} \mathrm{O}_{2}$ could be prevented by the addition of exogenous gangliosides. Flow cytometric analyses revealed that the pre-treatment of spermatozoa with GT1b micelles almost completely abolished $\mathrm{H}_{2} \mathrm{O}_{2}$ passage through the cell membrane (Figure 4). ${ }^{21}$ Reduced DNA fragmentation as a result of decreased cellular $\mathrm{H}_{2} \mathrm{O}_{2}$ suggests that the ganglioside GT1b acts as an inhibitor of $\mathrm{H}_{2} \mathrm{O}_{2}$ diffusion across the sperm membrane.

DNA integrity is also compromised during human sperm cryopreservation, which is routinely used in the management of human male infertility. The freezing/thawing process has been shown to generate sperm DNA damage including DNA fragmentation, base modifications, chromatin cross-linking and other alterations, although the exact mechanisms of the cryoinjury to sperm DNA have not been fully elucidated. ${ }^{4,15,71,72}$ Recent studies have shown that DNA frag-

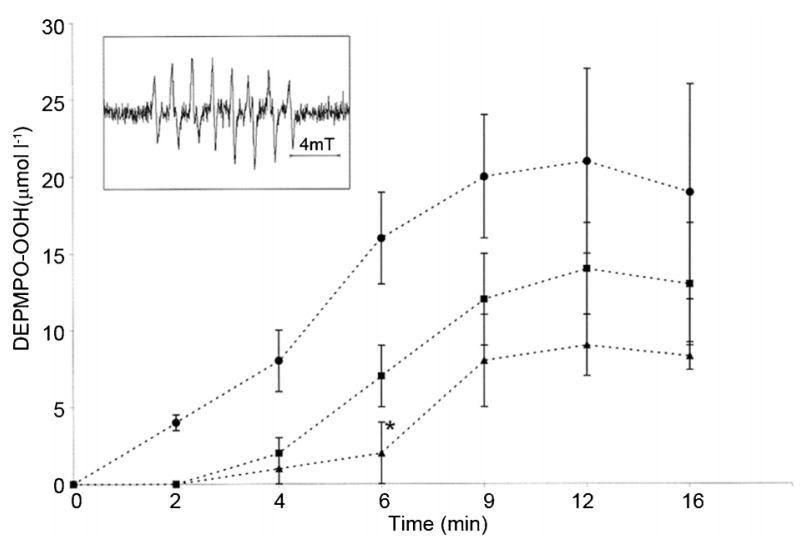

Figure 3 The influence of GT1b and GM1 micelles on the onset of superoxide production by PMA-activated PMNs $\left(5 \times 10^{6} \mathrm{ml}^{-1}\right)$ detected by EPR spectroscopy in the presence of the DEPMPO spin trap. The time-dependent superoxide anion generation by PMA-stimulated PMNs in the absence (filled circles) or presence (filled squares) of $100 \mu \mathrm{mol} \mathrm{I}{ }^{-1} \mathrm{GM} 1$ and GT1b (filled triangles). The data represent the mean \pm s.e.m. of five experiments. The typical experimental spectrum of the DEPMPO-OOH spin adduct is shown in the inset. $* P<0.005$ with GT1b compared to untreated cells. EPR, electron paramagnetic resonance; PMA, phorbol 12-myristate 13-acetate; PMN, polymorphonuclear neutrophils; DEPMPO, 5-diethoxyphosphoryl-5-methyl-1-pyrroline-N-oxide; DEPMPO-OOH, DEPMPO superoxide adduct.
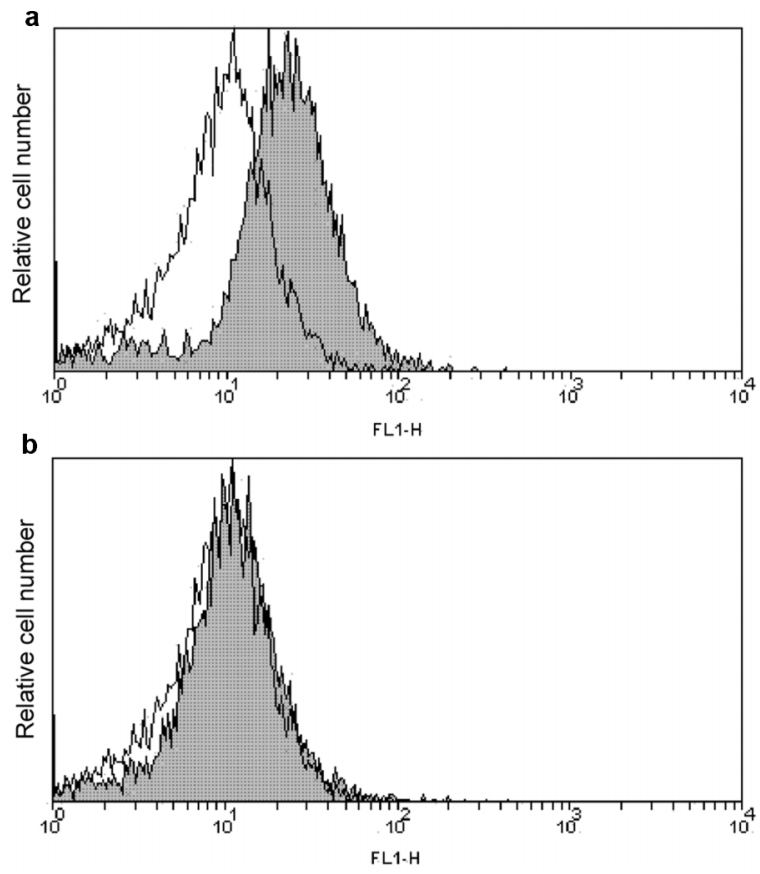

Figure 4 Flow cytometric analysis of $\mathrm{H}_{2} \mathrm{O}_{2}$ internalisation across the sperm membrane. The shaded histograms show the fluorescence intensity after the exogenous addition of $\mathrm{H}_{2} \mathrm{O}_{2}$ in the absence (a) or presence (b) of the ganglioside GT1b. The open histograms show the DCFH fluorescence of cells pre-incubated with GT1b only. A representative result of six separate experiments. PI-labelled dead cells were excluded from the analysis.

mentation can be primarily attributed to cryopreservation-induced ROS. ${ }^{73-75}$ In contrast to superoxide anion, the more stable $\mathrm{H}_{2} \mathrm{O}_{2}$ readily crosses the plasma membrane and is responsible for DNA oxidative damage. ${ }^{76}$ Because DNA integrity is essential for achieving fertilisation and enabling embryo development in assisted reproductive technologies, the preservation of DNA during cryostorage is of extreme importance. Several studies have examined antioxidative compounds that can protect sperm DNA from cryopreservation and thawing injuries. ${ }^{13,77}$

The cryopreservation-induced damage of the sperm membrane manifests as changes in the organisation and lipid composition of the membrane, leading to changes in sperm permeability. ${ }^{78,79}$ Several reports on mammalian sperm have demonstrated the protective capacity of phospholipid-based cryoprotectants. The most important role of phospholipids could be coating the cell membrane, resulting in reinforcement during cryostorage. ${ }^{80}$

Because the freezing/thawing process results in the loss of sperm surface hydrophobicity, ${ }^{81}$ one of the proposed mechanisms of sperm resistance to cryostorage-induced stress is its ability to shed hydrophilic lipids, thus increasing the hydrophobic properties of the membrane and enhancing sperm tolerance to cryopreservation. ${ }^{82}$ The exogenous addition of specific lipid compounds to the cell suspension can therefore be hypothesized to improve cell hydrophobicity and protect from cryopreservation-induced stress.

Such a protective effect of gangliosides has been confirmed in our recent report. We demonstrated that exogenously supplying both GM1 and GT1b at concentrations above the critical micellar concentration to the cryoprotective media protected human sperm DNA integrity during the freeze-thaw process (Figure 5). ${ }^{22}$

This could be due to ganglioside micelles associating with the sperm cell surface, thereby providing a physical diffusion barrier against 


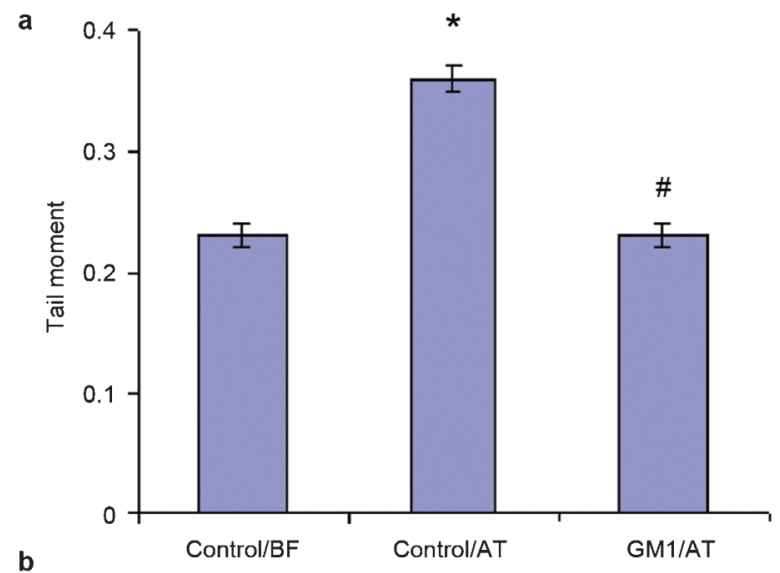

b

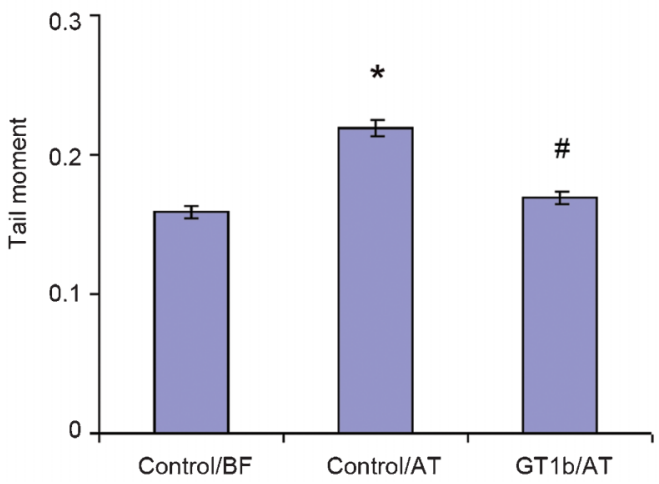

Figure 5 The effects of the monosialoganglioside GM1 and the trisialoganglioside GT1b on a Comet assay parameter (tail moment) in cryopreserved human spermatozoa. The data are presented (a) as mean \pm s.e. in the control samples and in the GM1-treated samples before freezing (BF) and after thawing (AT) $(n=8)$ and $(\mathbf{b})$ the mean \pm s.e. in the control samples and in the GT1b-treated samples before freezing (BF) and after thawing (AT) $(n=8)$. $* P<0.05$ vs. control cells before freezing. ${ }^{\#} P<0.05$ vs. control cells after thawing.

freeze-thaw damage. This is in agreement with our previous findings that exogenous GT1b acts as an inhibitor of $\mathrm{H}_{2} \mathrm{O}_{2}$ diffusion across the sperm membrane. ${ }^{21}$ We established that $43 \%$ and $73 \%$ of ganglioside micelles are removed in two washes, respectively, ${ }^{64}$ and they can therefore be assumed to simply adhere to the membrane surface through ion or hydrogen bonds. ${ }^{83}$ It appears that ganglioside micelles, which are loosely attached to the sperm cell membrane, increase membrane hydrophobicity during cryopreservation. Data on the effect of ganglioside micelles on neuronal cells have suggested a possible mediation of monomers, which escape from micelles during the micellemembrane interaction. ${ }^{83-85}$

Under our experimental conditions, ganglioside monomers from the surrounding cryoprotective media are not likely to have been inserted into the ganglioside-containing membrane lipid rafts because their insertion is known to be temperature-dependent and quite unlikely to occur at $4{ }^{\circ} \mathrm{C}$. ${ }^{86}$ At the present stage of research, these hypotheses require further investigations that rely on direct physicochemical methods.

\section{MECHANISMS OF SPERM PROTECTION BY GANGLIOSIDES}

The protective roles of gangliosides against ROS-induced changes in the sperm membrane that we observed appear to be associated with ganglioside amphiphilicity. The sugar moieties of gangliosides, specifically the sialic acid residues, are the basis of the observed protection against superoxide anions and iron-induced LPO. The protective mechanism of gangliosides against LPO is related to the ability of sialic acid to bind oppositely charged ferrous ions and reduce their concentration. The more pronounced effect of trisialoganglioside GT1b micelles than that of monosialoganglioside GM1 micelles can be attributed to their higher negative charge, which is the result of a higher sugar unit and sialic acid residue content. ${ }^{17}$ Similarly, the antioxidant effect of GT1b on free radical generation is greater than that of GM1, which has five sugar rings and only one sialic acid residue in contrast to GT1b, which has seven sugar rings and three sialic acid residues. ${ }^{25}$ As a consequence, GT1b micelles exhibit a higher negative charge due to a higher sialic acid content. Because of their greater hydrophilic repulsive contribution and larger number of hydrogen-bonding groups, GT1b molecules are prone to form smaller and more spherical aggregates that exhibit a two-fold lower aggregation number (176) than GM1 (301). ${ }^{25}$ The difference in their respective surface curvatures has been evidenced in their mean hydrodynamic diameters, which we found to be lower in GT1b micelles $(9 \mathrm{~nm})$ than in GM1 micelles $(11 \mathrm{~nm}) .{ }^{20}$ Based on reports demonstrating the importance of the GM1 and GT1b headgroups in the conformational properties of aggregate surfaces, the more pronounced effect observed in the presence of GT1b micelles compared to that of GM1 micelles can be ascribed to better steric shielding when smaller GT1b aggregates are adhered at the cell surface.

Another aspect of the protective activity of gangliosides determined in our studies is their potential to reduce $\mathrm{H}_{2} \mathrm{O}_{2}$ and cryopreservationinduced DNA damage. The ceramide moiety plays the predominant role in the ability of gangliosides to protect the sperm membrane. We have observed that GM1 and GT1b, having a different number of negatively charged sialic acid residues in the oligosaccharide chain and a similar hydrophobic moiety composition, have the same ability to protect sperm DNA from cryopreservation-induced damage. ${ }^{22}$ This demonstrates that the ceramide moiety, rather than the charges of the polar head groups, is involved in the reduction of DNA fragmentation. The presumed mechanism of spermatozoa protection during the freezing/thawing process might be explained by a hydrophobic interaction between the ceramide moiety of gangliosides and the lipid bilayer of the sperm cell membrane. Gangliosides are inserted into the membrane, intercalating with other hydrophobic components of the membrane and modulating the hydrophobic character of the sperm membrane, which is responsible for the increased tolerance to DNA fragmentation. It is possible that ganglioside micelles at the sperm surface increase the diffusion barrier against toxic oxidants, protecting against DNA damage, as shown in Figure 6.

\section{CONCLUDING REMARKS}

Due to their chemical and physical properties, gangliosides, which are sialic acid-containing glycophospholipids, are comparable with antioxidants whose in vitro and in vivo application has provided efficient protection of spermatozoa from oxidative stress-induced damage. The protective effect of ganglioside micelles against ROSinduced changes is believed to stem from their ability to scavenge free radicals and prevent their damaging effects.

Due to their multiple activities, gangliosides have advantages over many other antioxidant agents. The activities of ganglioside micelles include modulating superoxide anion generation by increasing the diffusion barrier for the membrane events responsible for signal translocation to the interior of the cell and inhibiting iron-catalysed hydroxyl radical formation through the iron chelation ability of gangliosides (Figure 6).

In addition, they are able to modulate the hydrophobic membrane properties that are responsible for better tolerance to DNA fragmentation 


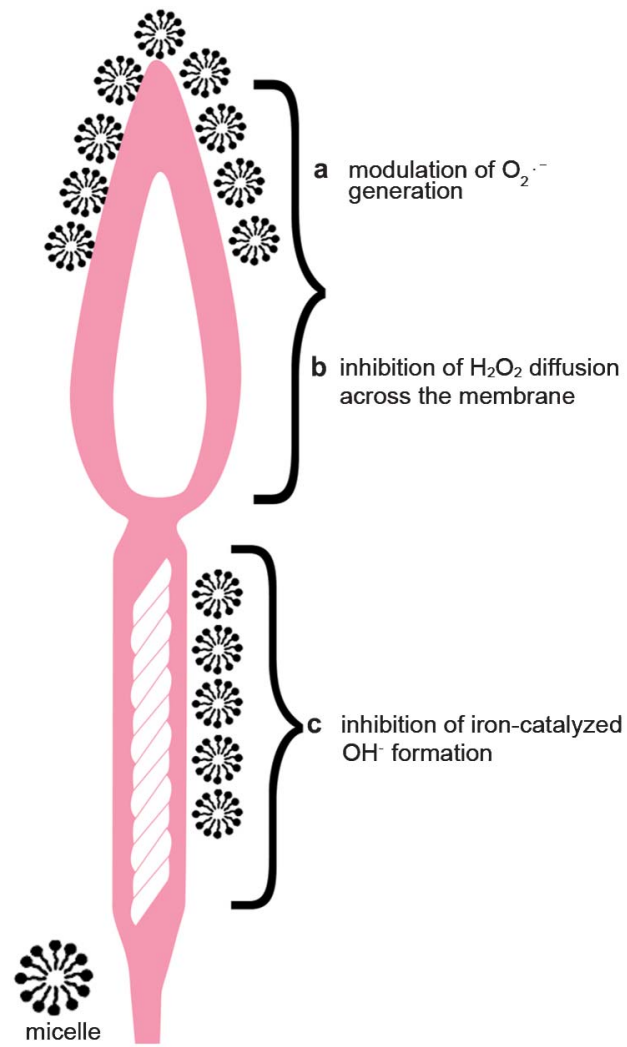

Figure 6 Exogenous ganglioside micelles attached to the outer part of the cell membrane increase membrane hydrophobicity and induce the following changes: (a) modulation of superoxide anion $\left(\mathrm{O}_{2} \cdot{ }^{-}\right)$generation by increasing the diffusion barrier for the membrane events responsible for signal translocation to the interior of the cell; (b) inhibition of $\mathrm{H}_{2} \mathrm{O}_{2}$ diffusion across the sperm membrane; (c) inhibition of iron-catalysed hydroxyl radical $(\mathrm{OH} \cdot)$ formation due to iron chelation.

following in vitro exposure to $\mathrm{H}_{2} \mathrm{O}_{2}$ and during the cryopreservation process. This last effect deserves special attention because it differentiates gangliosides from other antioxidants. While other antioxidants may reduce cryopreservation-induced DNA damage only to a certain extent, gangliosides are able to completely abolish DNA fragmentation.

The ganglioside activity that we observed in our in vitro experiments may open new possibilities for the development of cryostorage media to preserve DNA integrity, which will be essential for achieving optimal fertilisation and enabling embryo development in assisted conception therapy.

\section{COMPETING FINANCIAL INTERESTS}

The authors declare no competing financial interests.

\section{ACKNOWLEDGMENTS}

We gratefully acknowledge the contribution of Lovorka Perkovic in the editing and preparation of this manuscript and the support of the Ministry of Science, Education and Sports of the Republic of Croatia (No. 045-0000000-0174).

1 Tremellen K. Oxidative stress and male infertility-a clinical perspective. Hum Reprod Update 2008; 14: 243-58.

2 Aitken RJ, Roman SD. Antioxidant system and oxidative stress in the testes. Oxid Med Cell Longev 2008; 1: 15-24.
3 Aitken RJ, Koppers AJ. Apoptosis and DNA damage in human spermatozoa. Asian J Androl 2011; 3: 36-42.

4 Aitken RJ, de luliis GN. On the possible origins of DNA damage in human spermatozoa. Mol Hum Reprod 2010; 16: 3-13.

5 Lenzi A, Picardo M, Gandini L, Dondero F. Lipids of the sperm plasma membrane: from polyunsaturated fatty acids considered as markers of sperm function to possible scavenger therapy. Human Reprod Update 1996; 3: 246-56.

6 Jones R, Mann T, Sherins RJ. Peroxidative breakdown of phospholipids in human spermatozoa: spermicidal effects of fatty acid peroxides and protective action of seminal plasma. Fertil Steril 1979; 31: 531-7.

7 Aitken RJ, Harkiss D, Buckingham D. Relationship between iron-catalyzed lipid peroxidation potential and human sperm function. J Reprod Fertil 1993; 98: 257-65.

8 Aitken J, Fisher H. Reactive oxygen species generation and human spermatozoa: the balance of benefit and risk. BioEssays 1994; 16: 259-67.

9 Avendano C, Oehninger S. DNA fragmentation in morphologically normal spermatozoa: how much should we be concerned in the ICSI era? J Androl 2011; 32: 356-63.

10 Gavella M, Lipovac V, Marotti T. Effect of pentoxifyline on superoxide anion production by human sperm. Int J Androl 1991; 14: 320-7.

11 Hughes CM, Lewis SE, McKelvey-Martin VJ, Thompson W. The effects of antioxidant supplementation during Percoll preparation on human sperm DNA integrity. Hum Reprod 1998; 13: 1240-7

12 Li Z, Lin Q, Liu R, Xiao W, Liu W. Protective effects of ascorbate and catalase on human spermatozoa during cryopreservation. J Androl 2010; 31: 437-44.

13 Zini A, Al-Hathal A. Antioxidant therapy in male infertility: fact or fiction? Asian J Androl 2011; 13: 374-81.

14 Lombardo F, Sansone A, Romanelli F, Paoli D, Gandini L et al. The role of antioxidant therapy in the treatment of male infertility: an overview. Asian J Androl 2011; 13: 690-7.

15 Donnelly ET, Steele EK, McClure N, Lewis EM. Assessment of DNA integrity and morphology of ejaculated spermatozoa from fertile and infertile men before and after cryopreservation. Hum Reprod 2001; 16: 1191-9.

16 Martinez-Soto JC, de DiosHourcade J, Gutierrez-Adan A, Landeras JL, Gadea J. Effect of genistein supplementation of thawing medium on characteristics of frozen human spermatozoa. Asian J Androl 2010; 12: 431-41.

17 Gavella M, Lipovac V, Rakoš R, Čolak B. Reduction of oxidative changes in human spermatozoa by exogenous gangliosides. Andrologia 2005; 37: 17-24.

18 Kveder M, Rakoš R, Gavella M, Lipovac V, Pifat G et al. EPR investigation of cell membrane fluidity upon external oxidative stimulus. Appl Magn Reson 2004; 27: $77-86$

19 Gavella M, Kveder M, Lipovac V, Rakoš R, Pifat G. Trisialoganglioside GT1b prevents increase in sperm plasma molecular ordering induced by in vitro lipid peroxidation. I Androl 2005; 26: 724-31.

20 Gavella M, Kveder M, Lipovac V, Jurašin D, Filipović-Vinceković N. Antioxidant properties of ganglioside micelles. Free Radic Res 2007; 41: 1143-50.

21 Gavella M, Vrhovac-Garaj V, Lipovac V, Antica M, Gajski G et al. Ganglioside GT1b protects human spermatozoa from hydrogen peroxide-induced DNA and membrane damage. Int J Androl 2010; 33: 536-44.

22 Gavella M, Lipovac V, Garaj-Vrhovac V, Gajski G. Protective effect of ganglioside on DNA in human spermatozoa exposed to cryopreservation. J Androl 2012; 33: 101624.

23 Gore PI, Singh SP, Brooks DE. Composition of gangliosides from ovine testis and spermatozoa. Biochim Biophys Acta 1986; 876: 36-47.

24 Mack SR, Zaneveld CJ, Peterson RN, Hunt W, Russel LD. Characterization of human sperm plasma membrane: glycolipids and polypeptides. J Exp Zool 1987; 243: 33946.

25 Sonnino S, Mauri L, Chigorno V, Prinetti A. Gangliosides as components of lipid membrane domains. Glycobiology 2007; 17: 1R-13R.

26 Kotani M, Kawashima H, Ozawa T, Terashima T, Tai T. Differential distribution of major gangliosides in rat central nervous system detected by specific monoclonal antibodies. Glycobiology 1993; 3: 137-46.

27 Svennerholm L. Chromatografic separation of human brain gangliosides. J Neurochem 1963; 10: 613-23

28 Vasudevan SJ, Balaji PV. Comparative analysis of ganglioside conformations by MD stimulations: implications for specific recognition by proteins. I $\mathrm{Mol}$ Struc (Theochem) 2002; 583: 215-32.

29 Malisan F, Testi R. GD3 in cellular ageing and apoptosid. Exp Gerontol 2002; 37: 1273-82.

30 Hakomori S. Structure, organization and function of glycosphingolipids in membrane. Curr Opin Hematol 2003; 1: 16-24.

31 Simons K, Ikonen E. Functional rafts in cell membranes. Nature 1997; 387: 569-72.

32 Simons K, Tooimre D. Lipid rafts and signal transduction. Nat Rev Mol Cel/ Bio/2000; 1: 31-9.

33 Maulik N, Das DK, Gogineni M, Cordis DA, Avrova N et al. Reduction of myocardial ischemic reperfusion injury by sialylated glycosphingolipids, gangliosides. J Cardiovasc Pharmacol 1993; 22: 74-81.

34 Bucolo C, Lin LR, Dang L, Giblin FJ, Reddy VN. The effect of ganglioside on oxidationinduced permeability changes in lens and in epithelial cells of lens and retina. Exp Eye Res 1994; 58: 697-704.

35 Sergent O, Pereira M, Belhomme C, Chevanne M, Huc L et al. Role for membrane fluidity in ethanol-induced oxidative stress of primary rat hepatocytes. J Pharmacol Exp Ther 2005; 313: 104-11. 
36 Tyurina YY, Tyurin VA, Avrova NF. Ganglioside GM1 protects CAMP $3^{\prime} 5^{\prime}$ : phosphodiesterase from inactivation caused by lipid peroxidation in brain synaptosomes of rats. Mol Chem Neuropathol 1993; 19: 205-17.

37 Avrova N, Zakharova IO, Tyurin VA, Tyurina YY, Gamaley IA et al. Different metabolic effects of ganglioside GM1 in brain synaptosomes and phagocytic cells. Neurochem Res 2002; 27: 751-9.

38 Fighera MR, Bonini JS, Frussa-Filho R, Dutra-Filho CS, Hagen ME et al. Monosialoganglioside increases catalase activity in cerebral cortex of rats. Free Radic Res 2004; 38: 495-500.

39 Mahadik SP, Makar TK, Murthy JN, Ortiz A, Wakade CG et al. Temporal changes in superoxide dismutase, glutathione peroxidase, and catalase levels in primary and periishemic tissue. Monosialoganglioside (GM1) treatment effects. $\mathrm{Mol}$ Chem Neuropathol 1993; 18: 1-14.

40 Avrova NF, Sokolova TV, Vlasova YA, Zakharova IO, Furaev V et al. Protective and antioxidative effects of GM1 ganglioside in $\mathrm{PC} 12$ cells exposed to hydrogen peroxide are mediated by Trk tyrosine kinase. Neurochem Res 2010; 35: 85-98.

41 Yamamoto H, Mohanan PV. Ganglioside GT1b and melatonin inhibit brain mitochondrial DNA damage and seizures induced by kainic acid in mice. Brain Res 2003; 964: 100-6.

42 Gorria M, Huc L, Sergent O, Rebillard A, Gaboriau F et al. Protective effect of monosialoganglioside GM1 against chemically induced apoptosis through targeting of mitochondrial function and iron transport. Biochem Pharmacol 2006; 72: 1343 53.

43 Oshio S, Kaneko R, lizuka R, Mohri H. Sialic acid in purified human semen. Arch Androl 1987; 18: 225-30.

44 Buttke DE, Nelson JL, Schlegel PN, Hunnicutt GR, Travis AJ. Visualization of GM with cholera toxin in live epididymal versus ejaculated bull, mouse and human spermatozoa. Biol Reprod 2006; 74: 889-95.

45 Cross NL. Reorganization of lipid rafts during capacitation of human sperm. Biol Reprod 2004; 71: 1367-73.

46 Selveraj V, Buttke DE, Asano A, McElwee JL, Wolff CA et al. GM 1 dynamics as a marker for membrane changes associated with the process of capacitation in murine and bovine spermatozoa. J Androl 2007; 28: 588-99.

47 Nixon B, Aitken RJ. The biological significance of detergent-resistant membranes in spermatozoa. J Reprod Immunol 2009; 83: 8-13.

48 Jones R, Howes E, Dunne PD, James P, Bruckbauer A et al. Tracking diffusion of GM 1 gangliosides and zona pellucida binding molecules in sperm plasma membranes following cholesterol efflux. Develop Biol 2010; 339: 398-406.

49 Kawano N, Yoshida K, Iwamoto T, Yoshida M. Ganglioside GM1 mediates decapacitation effects on SVS2 on murine spermatozoa. Biol Reprod 2008; 79 1153-1159.

50 Aitken RJ, Harkiss D, Buckingham DW. Analysis of lipid peroxidation mechanisms in human spermatozoa. Mol Reprod Dev 1993; 35: 302-15.

51 Alvarez JG, Storey BT, Evidence that membrane stress contributes more than lipid peroxidation to sublethal cryodamage in cryopreserved human sperm: glycerol and other polyols as sole protectant. J Androl 1992; 13: 199-209.

52 Storey BT. Biochemistry of the induction and prevention of lipoperoxidative damage in human spermatozoa. Mol Hum Reprod 1997; 3: 203-13.

53 Gomez E, Irvine DS, Aitken RJ. Evaluation of a spectrophotometric assay for the measurement of malondialdehyde and 4-hydroxyalkenals in human spermatozoa: relationships with semen quality and sperm function. Int J Androl 1998; 21: 81-94.

54 Ball B, Vo A. Detection of lipid peroxidation in equine spermatozoa based upon the lipophilic fluorescent dye $C_{11}$-BODIPY581/591. J Androl 2002; 23: 259-69.

55 Giraud MN, Motta C, Boucher D, Grizard G. Membrane fluidity predicts the outcome of cryopreservation of human spermatozoa. Hum Reprod 2000; 15: 2160-4.

56 Ochsendorf FR, Rinne D, Fuchs J, Such P, Zimmer G. Electron paramagnetic resonance spectroscopy for the investigation of the fluidity of human spermatozoa plasma membranes: a feasibility study. Andrologia 2000; 32: 169-77.

57 Force A, Grizard G, Giraud MN, Motta C, Sion B et al. Membrane fluidity and lipid content of human spermatozoa selected by swim-up method. Int J Androl 2001; 24 327-34.

58 Nivsakar M, Patel RY, Mokal R. Modulation of sperm membrane conformation by pentoxifylline in oligospermia: a biophysical investigation of sperm membrane in vitro. Biochem Biophys Res Commun 1996; 225: 791-5.

59 Christova Y, James PS, Jones R. Lipid diffusion in sperm plasma membranes exposed to peroxidative injury from oxygen free radicals. Mol Reprod Dev 2004; 68: 365-72.

60 Bondy SC, McKee M, Davoodbhoy YM. Prevention of chemically induced changes in synaptosomal membrane order by ganglioside GM1 and $\alpha$-tocopherol. Biochim Biophys Acta 1990; 1026: 213-9.
61 Garcia JJ, Reiter RJ, Karbownik M, Calvo JR, Ortiz GG et al. N-acetylserotonin suppresses hepatic microsomal membrane rigidity associated with lipid peroxidation. Eur J Pharmacol 2001; 428: 169-75.

62 Kwenang A, Kroos MJ, Foster JF, van Eijk HG. Iron, ferritin and cooper in seminal plasma. Hum Reprod 1987; 2: 387-8.

63 Sokolova TV, Furaev VV, Victorov V, Andreeva NA, Avrova NF. Stimulation by gangliosides of viability of rat brain neurons and of neuronal PC12 cell line under conditions of oxidative stress. J Evol Biochem Physiol 2005; 41: 415-23.

64 Gavella M, Kveder M, Lipovac V. Modulation of ROS production in human leukocytes by ganglioside micelles. Braz J Med Biol Res 2010; 43: 942-9.

65 Henkel RR. Leukocytes and oxidative stress: dilemma for sperm function and male fertility. Asian J Androl 2011; 13: 43-52.

66 Henkel R. ROS and semen quality. In: Agarwal A, Aitken RJ, Alvarez JG, editors Studies on Men's Health and Fertility. New York: Humana Press; 2012. p301.

67 Meyers SA. Cryostorage and oxidative stress in mammalian spermatozoa. In: Agarwa A, Aitken RJ, Alvarez JG, editors. Studies on Men's Health and Fertility. New York: Humana Press; 2012. p41.

68 Bedard K, Krause KH. The Nox family of ROS-generating NADPH oxidases: physiology and pathophysiology. Physiol Rev 2007; 87: 245-313.

69 Russo A, Troncoso N, Sanchez F, Garbarino JA, Vanella A. Propolis protects human spermatozoa from DNA damage caused by benzo(a)pyrene and exogenous reactive oxygen species. Life Sci 2006; 78: 1401-6.

70 Sierens J, Hartley JA, Campbell MJ, Leathem AJ, Woodside JV. In vitro isoflavone supplementation reduces hydrogen peroxide induced DNA damage in sperm. Teratog Carcinog Mutagen 2002; 22: 227-34.

71 Aitken RJ, Gordon E, Harkiss D, Twigg JP, Milne P et al. Relative impact of oxidative stress on the functional competence and genomic integrity of human spermatozoa. Biol Reprod 1998; 59: 1037-46.

72 Hammadeh ME, Askari AS, Georg T, Rosenbaum P, Schmidt W. Effect of freezethawing procedure on chromatin stability, morphological alteration and membrane integrity of human spermatozoa in fertile and subfertile men. Int J Androl 1999; 22 155-62.

73 Tamburino L, Marchiani S, Marion FE, Natali I, Cambi M et al. Mechanisms and clinical correlates of sperm DNA damage. Asian J Androl 2012; 14: 24-31.

74 Thomson LK, Fleming SD, Aitken RJ, de Iuliis GN, Zieschang JA et al. Cryopreservation-induced human sperm DNA damage is predominantly mediated by oxidative stress rather than apoptosis. Hum Reprod 2009; 24: 2061-70.

75 Zribi N, Chakroun NF, El Euch H, Gargouri J, Bahloul A et al. Effects of cryopreservation on human sperm deoxyribonucleic acid integrity. Fertil Steril 2010; 93: 159-66.

76 Baumber J, Ball BA, Linfor JJ, Meyers SA. Reactive oxygen species and cryopreservation promote DNA fragmentation in equine spermatozoa. J Androl 2003; 24: 621-8.

77 Zini A, San Gabriel M, Baazeem A. Antioxidants and sperm DNA damage: a clinical perspective. J Assist Reprod Genet 2009; 26: 427-32.

78 James PS, Wolfe CA, Mackie A, Ladha S, Prentice A et al. Lipid dynamics in the plasma membrane of fresh and cryopreserved human spermatozoa. Hum Reprod. 1999; 14: 1827-32.

79 James PS, Hennessy C, Berge T, Jones R. Compartmentalisation of the sperm plasma membrane; a FRAP, FLIP and SPFI analysis of putative diffusion barriers on the sperm head. J Cell Sci 2004; 117: 6485-95.

80 Ricker JV, Linfor JJ, Delfino WJ, Kysar P, Scholz EL et al. Equine sperm membrane phase behavior: the effects of lipid-based cryoprotectants. Biol Reprod 2006; 74 359-65.

81 Ollero M, Perez-Pe R, Muino-Blanco T, Cebrian-Perez A. Improvement of ram sperm cryopreservation protocols assessed by sperm quality parameters and heterogeneity analysis. Cryobiology 1998; 7: 1-12.

82 Chakrabarty J, Banerjee D, Pal J, Ghosh A, Majumder GC. Shedding off specific lipid constituents from sperm cell membrane during cryopreservation. Cryobiology 2007; 54: 27-35.

83 Toffano G, Benvegnu D, Bonneti AC, Facci AC, Leon A et al. Interactions of GM1 ganglioside with crude rat brain neuronal membranes. J Neurochem 1980; 35 861-6.

84 Kanda S, Inoue K, Nojima S, Utsumi H, Wiegandt H. Incorporation of a ganglioside and a spin-labeled ganglioside analogue into cell and liposomal membranes. $J$ Biochem 1982; 91 : 2095-8.

85 Leskawa KC, Erwin RE, Leon A, Toffano G, Hogan EL. Incorporation of exogenous ganglioside GM1 into neuroblastoma membranes: inhibition by calcium ion dependence upon membrane protein. Neurochem Res 1989; 14: 547-54.

86 Saqr HE, Pearl DK, Yates AJ. A review and predictive models of ganglioside uptake by biological membranes. J Neurochem 1993: 61: 395-410. 